\title{
Transverse spin and transverse momentum structure of the nucleon from the COMPASS experiment
}

\author{
Federica Sozzi ${ }^{1, a}$ on behalf of the COMPASS Collaboration \\ ${ }^{1}$ Trieste Section of INFN, 34127 Trieste, Italy
}

\begin{abstract}
COMPASS is a fixed target experiment at the CERN SPS M2 beam line, taking data since year 2002. Part of the physics programme is dedicated to the study of the transverse spin and transverse momentum structure of the nucleon, by means of semi-inclusive deeply inelastic lepton scattering (SIDIS). For these measurements, data have been collected using transversely polarised proton $\left(\mathrm{NH}_{3}\right)$ and deuteron $\left({ }^{6} \mathrm{LiD}\right)$ targets. A selection of the results on the azimuthal asymmetries measured is presented, with particular focus on the most recent measurements from the data collected in 2007 and 2010 with the proton target. These results exhibit clear signals for the Collins asymmetry, interpreted as a convolution of a non-zero transversity PDF and Collins fragmentation function, and for the Sivers asymmetry which is related to the Sivers function, the most famous and discussed of the TMD PDFs. The data collected with the ${ }^{6} \mathrm{LiD}$ target, have also been recently analysed to search for the azimuthal modulations in the production of hadrons which are expected to be present in the unpolarised part of the SIDIS cross-section. The azimuthal hadron asymmetries, which are related to the Boer-Mulders TMD PDF, show strong and somewhat puzzling kinematical dependencies.
\end{abstract}

\section{Introduction}

The description of the partonic structure of the nucleon is one of the central problems of hadronic physics. In recent years considerable theoretical and experimental progress has been made [1] and the relevance of the quark transverse spin and transverse momentum has been clearly assessed. In the present theoretical framework, eight transverse momentum dependent parton distribution functions (TMD PDFs) are required at leading twist for each quark flavor. They describe all possible correlations between the transverse momentum and spin of the quarks, and the spin of the nucleon. When integrating over the quark transverse momentum, five of these functions vanish, while three of them give the well known number, helicity and transversity distribution functions. Among these last three functions, the transversity distribution is the analogous of the helicity PDFs in the case of transversely polarised nucleons, and experimentally it is the least known. On the experimental side, several complementary processes are being, and will be, studied to access the TMD PDFs, namely transversely polarised hard proton-proton scattering, Drell-Yan processes, and SIDIS. This last channel is today the major source of information. The main advantage of the SIDIS channel is that TMD effects are not mixed: at variance with hadroproduction, the TMD effects generate different azimuthal modulations

\footnotetext{
a e-mail: federica.sozzi@ts.infn.it
} 
in the SIDIS cross section, which can be separately explored and extracted from the same data. The modulations are the combination of two angles, $\phi_{S}$ and $\phi_{h}$, the azimuthal angle of the initial nucleon spin and of the produced hadron momentum. The angles are defined with respect to the direction of the virtual photon, defining the $\mathrm{z}$ axis of the so called Gamma Nucleon System, in which the $\mathrm{xz}$ plane is defined by the lepton scattering plane. The modulation amplitudes are different structure functions, proportional to convolutions of the TMD PDFs and fragmentation functions (FFs). An important part of the experimental programme of the COMPASS experiment consists in the study of the nucleon structure. In the following a summary of the main measurements related to the transverse structure of the nucleon are presented.

\section{The COMPASS experiment}

COMPASS [2] (COmmon Muon and Proton Apparatus for Structure and Spectroscopy) is a fixed target experiment at the CERN SPS taking data since 2002. SIDIS data have been collected using a 160 $\mathrm{GeV}$ longitudinally polarised muon beam and longitudinally or transversely polarised proton $\left(\mathrm{NH}_{3}\right)$ and deuteron $\left({ }^{6} \mathrm{LiD}\right)$ targets. The spectrometer comprises a variety of different tracking detectors, and allows to detect charged tracks in a broad momentum and angular range. Calorimeters, muon filters and a gas radiator RICH detector are available for particle identification. In particular, the RICH detector allows to identify particles as pions and kaons in the momentum range from 3 and $9 \mathrm{GeV} / \mathrm{c}$, respectively, and up to $50 \mathrm{GeV} / \mathrm{c}$.

\section{Transverse azimuthal spin asymmetries}

To access the target polarization dependent structure functions, suitable spin asymmetries are built comparing the azimuthal distribution of hadrons produced in reactions on targets with opposite spin orientation. For the transverse target polarization, there are 4 leading twist asymmetries:

- $A_{U T}^{\sin \left(\phi_{h}+\phi_{S}\right)} \propto h_{1} \otimes H_{1}^{\perp}$ (Collins asymmetries);

- $A_{U T, T}^{\sin \left(\phi_{h}-\phi_{S}\right)} \propto f_{1 T}^{\perp} \otimes D_{1}$ (Sivers asymmetries);

- $A_{U T}^{\sin \left(3 \phi_{h}-\phi_{S}\right)} \propto h_{1 T}^{\perp} \otimes H_{1}^{\perp}$;

- $A_{L T}^{\cos \left(\phi_{h}-\phi_{S}\right)} \propto g_{1 T} \otimes D_{1}$;

where the first and the second subscripts indicate the beam and target polarization (U unpolarised, L longitudinal and T-transverse), the third subscript the virtual photon polarization, and the superscript the azimuthal modulation. As can be seen on the right-hand side of the above equations, each asymmetry gives access to a different PDF, convoluted with the unpolarised FF, $D_{1}$, or the Collins FF, $H_{1}^{\perp}$. The latter describes the correlation between the quark spin and the momentum of the produced hadron. The PDFs $h_{1}, f_{1 T}^{\perp}, h_{1 T}^{\perp}$ and $g_{1 T}$ are, respectively, the transversity, Sivers, pretzelosity, and "worm-gear" PDFs.

\subsection{Collins asymmetries}

The Collins asymmetries for charged pions and kaons from all the collected proton data are shown in fig. 1 , as a function of the Bjorken variable $x$, the fractional energy of the hadron $z$, and the hadron transverse momentum $p_{T}^{h}$. The pions asymmetries are very similar to the published unidentified hadron asymmetries [3]: at small $x$ the asymmetries are compatible with zero, while in the valence region the asymmetries show an increasing signal, of opposite sign for $\pi^{+}$and $\pi^{-}$. This naively indicates 

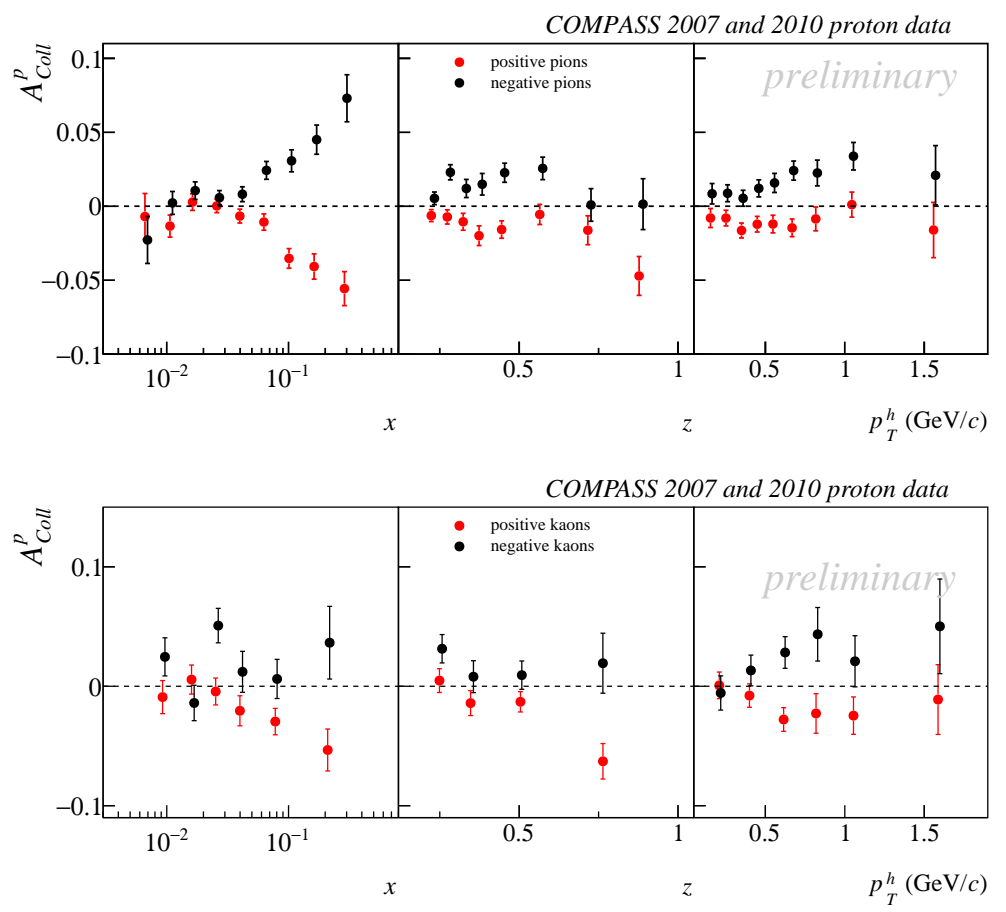

Figure 1. The Collins asymmetries for pions (top) and kaons (bottom) on proton measured by COMPASS, as a function of $x, z$ and $p_{T}^{h}$.

that the unfavored and favored Collins FF have opposite sign. The results for charged kaons, even if affected by larger error bars, show a similar trend: in particular the $\mathrm{K}^{+}$asymmetry has a negative trend in the valence region, and the $\mathrm{K}^{-}$one is positive on average. It is interesting to compare these results with the other existing measurements on a proton target, from the HERMES experiment [4]. In the large $x$ region common to both experiments, the measurements are in good agreement, a non obvious result due to the different kinematic range covered: in the last $x$ bins, the COMPASS $Q^{2}$ is larger of a factor 2-3 wrt HERMES one.

All the asymmetries measured by COMPASS on deuteron $[5,6]$ are found to be compatible with zero, also for kaons. This result is interpreted as a cancellation between the $\mathrm{u}$ and $\mathrm{d}$ quark contribution in an isoscalar target.

\subsection{Sivers asymmetries}

The Sivers asymmetries measured by COMPASS for pions and kaons on a proton target are shown in fig. 2. Also in this case the pion asymmetries are very similar to the recently published unidentified hadron asymmetries [7]. The asymmetries for negative pions and kaons are compatible with zero, while for positive pions and kaons there is an evidence for a positive signal extending overall the measured $x$ region, and increasing with $z$. Very intriguing is the fact that the $K^{+}$signal is larger than that of $\pi^{+}$, hinting at a possible not negligible role of the sea quarks. At variance with the Collins 

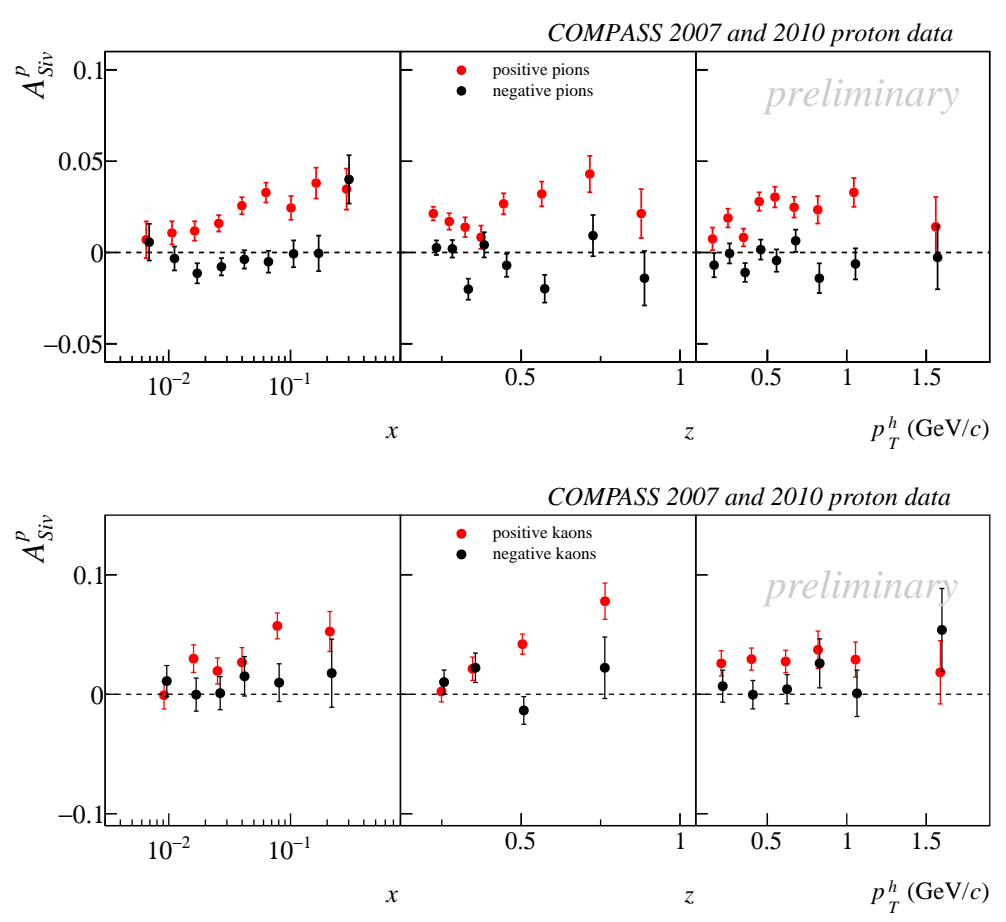

Figure 2. The Sivers asymmetries for pions (top) and kaons (bottom) on proton measured by COMPASS, as a function of $x, z$ and $p_{T}$.

case, at large $x$ the COMPASS Sivers asymmetries are smaller by about a factor of two with respect to HERMES's ones [8], giving insights of the $Q^{2}$ evolution of the TMD functions.

Results for these asymmetries on a deuterium target are also available [5, 6], allowing a handle on the d-quark PDF. All the asymmetries measured on deuterium have been found compatible with zero, interpreted as a cancellation of the $u$ and d quark contributions in the isocalar deuterium.

\subsection{Other transverse spin asymmetries}

The $A_{U T}^{\sin \left(3 \phi_{h}-\phi_{S}\right)}$ asymmetry, giving access to the pretzelosity PDF, has been found compatible with zero both on deuteron and proton targets. Regarding the $A_{L T}^{\cos \left(\phi_{h}-\phi_{S}\right)}$ asymmetries, while the results on the deuteron target are compatible with zero, the proton results show a small positive signal with an increasing trend in $x$. A similar trend is also present in HERMES preliminary results and in JLab E06-00 results on neutron.

\section{Unpolarised azimuthal asymmetries}

The data collected with the ${ }^{6} \mathrm{LiD}$ target, suitably mixed up to cancel possible target polarization effects, have also been analysed to search for the azimuthal modulations in the production of hadrons which are expected to be present in the unpolarised part of the SIDIS cross-section. There are three independent modulations in the hadron azimuthal distribution: 

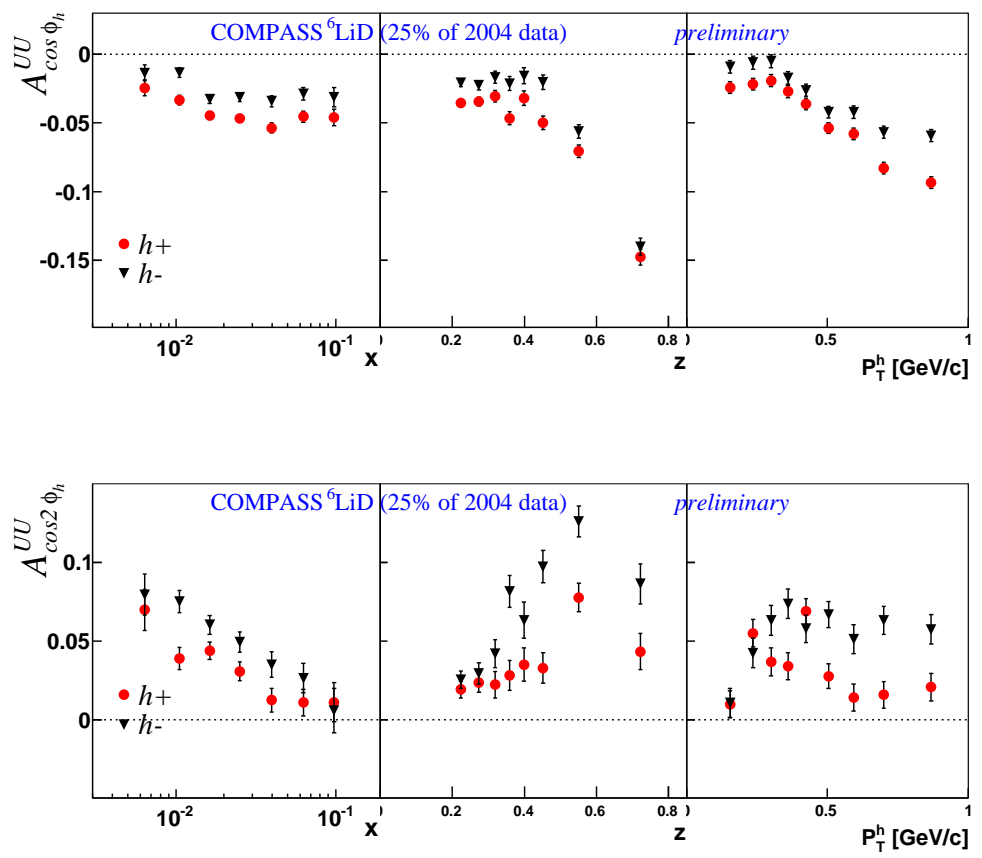

Figure 3. The Cahn and $A_{U U}^{\cos \left(2 \phi_{h}\right)}$ asymmetries for positive and negative hadrons on ${ }^{6} \mathrm{LiD}$ target measured by COMPASS, as a function of $x, z$ and $p_{T}$.

- $A_{L T}^{\sin \left(\phi_{h}\right)}$ : this is a higher twist effect proportional to the beam polarization, and has no clear interpretation in the parton model;

- $A_{U U}^{\cos \left(\phi_{h}\right)}$ (Cahn asymmetries): this asymmetry is due mainly to the so called Cahn effect, a kinematical effect due to the quark transverse momentum. A higher twist contribution is also expected by the Boer-Mulders TMD PDF;

- $A_{U U}^{\cos \left(2 \phi_{h}\right)}$ : Boer-Mulders TMD PDF is expected to give a leading twist contribution, while the Cahn effect is suppressed by a factor $1 / \mathrm{Q}$.

The results for the Cahn asymmetries are shown in the top part of fig. 3, for positive and negative unidentified hadrons. A large signal over all the $x$ range is visible, as well as a strong $z$ dependence, for $z$ above 0.5. A strong dependence with the kinematical variables is also present for the $A_{U U}^{\cos \left(2 \phi_{h}\right)}$ asymmetries, shown in the bottom part of fig 3 . In this case there is a large signal at small $x$, decreasing with $x$. The strong dependencies, particularly on $p_{T}^{h}$, are difficult to describe in the present phenomenological framework. In order to understand the kinematical dependencies of these asymmetries, a multi-dimensional analysis has been done, binning simultaneously in $x, z$ and $p_{T}^{h}$ [9]. This analysis allows to locate more precisely the different trends. 


\section{Conclusion}

The COMPASS experiment has investigated transverse spin and TMD effects using deuterium and proton targets. A full set of results on the Collins and Sivers asymmetries, on pions and kaons is provided. While the results on the deuterium target are compatible with zero, there are interesting non vanishing effects on protons, to be further investigated on the already collected data through a multi-dimensional analysis. The azimuthal unpolarised asymmetries on deuterium are different from zero, showing complex and interesting behavior in the kinematical variables. New measurements on a liquid hydrogen target are foreseen in the second phase of COMPASS, in parallel to the DVCS data taking starting from 2016.

\section{References}

[1] V. Barone, F. Bradamante and A. Martin, Prog. Part. Nucl. Phys. 65, 267 (2010)

[2] COMPASS Collaboration, Ageev E. S. et al., Nucl. Instr. Meth. A, 577, 455 (2007)

[3] COMPASS Collaboration, Adolph C. et al., Phys. Lett. B, 717, 376 (2012)

[4] HERMES Collaboration, Airapetian A.et al.,Phys. Lett. B, 693, 11 (2010)

[5] COMPASS Collaboration, Ageev E. S. et al., Phys. Lett. B, 765, 127 (2007)

[6] COMPASS Collaboration, Alekseev M. et al., Phys. Lett. B, 673, 127 (2009)

[7] COMPASS Collaboration, Adolph C. et al., Phys. Lett. B, 717, 383 (2012)

[8] HERMES Collaboration, Airapetian A. et al., Phys. Rev. Lett., 103, 152002 (2009)

[9] G.Sbrizzai for the COMPASS Collaboration, QNP2012 6th International Conference on Quarks and Nuclear Physics, Palaiseau, France, April 16-20, 2012 